\title{
Development of SEM Equipped with Superconductor X-ray Detector Toward Chemical State Nanoimaging for CFRP/adhesive Boundary
}

Go Fujii ${ }^{1}$, Wenqin Peng ${ }^{1}$, Masahiro Ukibe ${ }^{1}$, Shigetomo Shiki ${ }^{1}$, Naoko Yamazaki ${ }^{2}$, Koichi Hasegawa ${ }^{2}$, Kiyoka Takagi ${ }^{2}$ and Masataka Ohkubo ${ }^{1}$

${ }^{1}$ National Institute of Advanced Industrial Science and Technology, Tsukuba, Ibaraki, Japan, ${ }^{2}$ Mitsubishi Heavy Industries, Ltd., Nagoya, Aichi, Japan

Carbon fiber reinforced plastics (CFRPs) exhibit a high strength-to-weight ratio and a toughness better than metals. Because of such superior mechanical properties, CFRP composite structures are becoming popular in aircraft and automobile industries. The lightweight structure brings many benefits like a good fuel efficient, which is the central issue in transportation. The CFRP composite structures such as wing spars and fuselage components of the aircraft are fabricated by bonding the CFRP parts with adhesive and bolting. However, since the bolting causes a mechanical strength degradation and also the bolting process is time and manpower consuming, the boltless CFRP composite structures are required. In order to realize the boltless structure, it is necessary to understand the adhesion mechanism to estimate theoretically the mechanical strength of the boltless structure, and for that purpose, it is important to analyze chemical bonding states at those boundaries. However, chemical bonding state analysis at the boundaries has not been realized so far, since the analysis at a spatial resolution of less than $10 \mathrm{~nm}$ should be performed.

One candidate of nanoimaging techniques of the chemical bonding state is a combination of X-ray emission spectroscopy (XES) using X-ray detectors with high energy resolution and a nanoscale electron beam produced by SEM. By using conventional energy-dispersive X-ray detectors such as silicon drift detectors (SDDs) or $\mathrm{Si}(\mathrm{Li})$ detectors, elemental nanoimaging of the boundary can be demonstrated, although the XES is impossible because the energy resolution of the detectors was more than $50 \mathrm{eV}$ for soft X-rays [1]. On the other hands, wave-dispersive X-ray spectrometer with a diffraction grating can perform the XES of various bulk materials due to high energy resolution of less than $1 \mathrm{eV}[2],[3]$. However, epoxy materials such as the resin and adhesive used in the CFRP composites can't be performed the XES by the spectrometer due to those low throughput because the chemical bonding state of the epoxy is easily affected by electron beam irradiation.

In contrast, energy-dispersive X-ray detectors based on superconducting-tunnel-junctions (STJs) have best energy resolution ( $\triangle \mathrm{E}$ ) of $4.1 \mathrm{eV}$ for $400 \mathrm{eV}$ soft X-rays[4] and can distinguish difference in shape of $\mathrm{X}$-ray emission spectrum of $\mathrm{C}-K$ between different carbon-based materials[5]. In addition, our 100-pixel STJ array detector was realized relatively large detection area of about $1 \mathrm{~mm}^{2}$ and high counting rate capability of $>200 \mathrm{kcps}$, whose properties make it possible to perform high-throughput analysis. In this work, we have developed a SEM equipped with the 100-pixel STJ array, which is abbreviate as SC-SEM hereafter. In order to demonstrate performances of the SC-SEM as a first step, we have performed an elemental mapping of a boundary between the resin and adhesive with a high spatial resolution.

Figure 1 shows a picture of the SC-SEM. The SC-SEM consisted of a Schottky Field Emission SEM and the 100-pixel STJ array X-ray detector. The STJ array was cooled to $0.3 \mathrm{~K}$ on a cold stage of a cryogenfree helium-3 cryostat. X-rays emitted from the sample by the electron beam were detected by the STJ array via a polycapillary X-ray lens [6]. In order to improve the collection efficiency, the X-ray lens was installed in the system. The collection efficiency of the SC-SEM was about $10 \mathrm{mSr}$, which was about 1/10 times smaller than that of the SDDs [7]. 
Figure 2 shows SEM and 2D elemental mapping images of the boundary between the resin and adhesive. The resin and adhesive were TGDDM and DGEBA cured with DDS, respectively. The images were obtained in the acceleration-voltage of $3 \mathrm{kV}$ and probe current of $0.2 \mathrm{nA}$. In the SEM image, a boundary between the resin and adhesive having a similar composition can be clearly observed. In the $2 \mathrm{D}$ elemental mapping images, intensity differences of $\mathrm{C}-K \alpha, \mathrm{N}-K \alpha$, and $\mathrm{O}-K \alpha$ between the resin and adhesive were observed, and the boundary can be clearly identified. The spatial resolution of the elemental mapping was about $50 \mathrm{~nm}$. By further reducing the acceleration-voltage, the spatial resolution will be improved. In the future, by the XES in the SC-SEM, we will realize the nanoimaging of the chemical bounding state of the boundary.

This work was supported by Innovative Science and Technology Initiative for Security, ATLA, Japan.

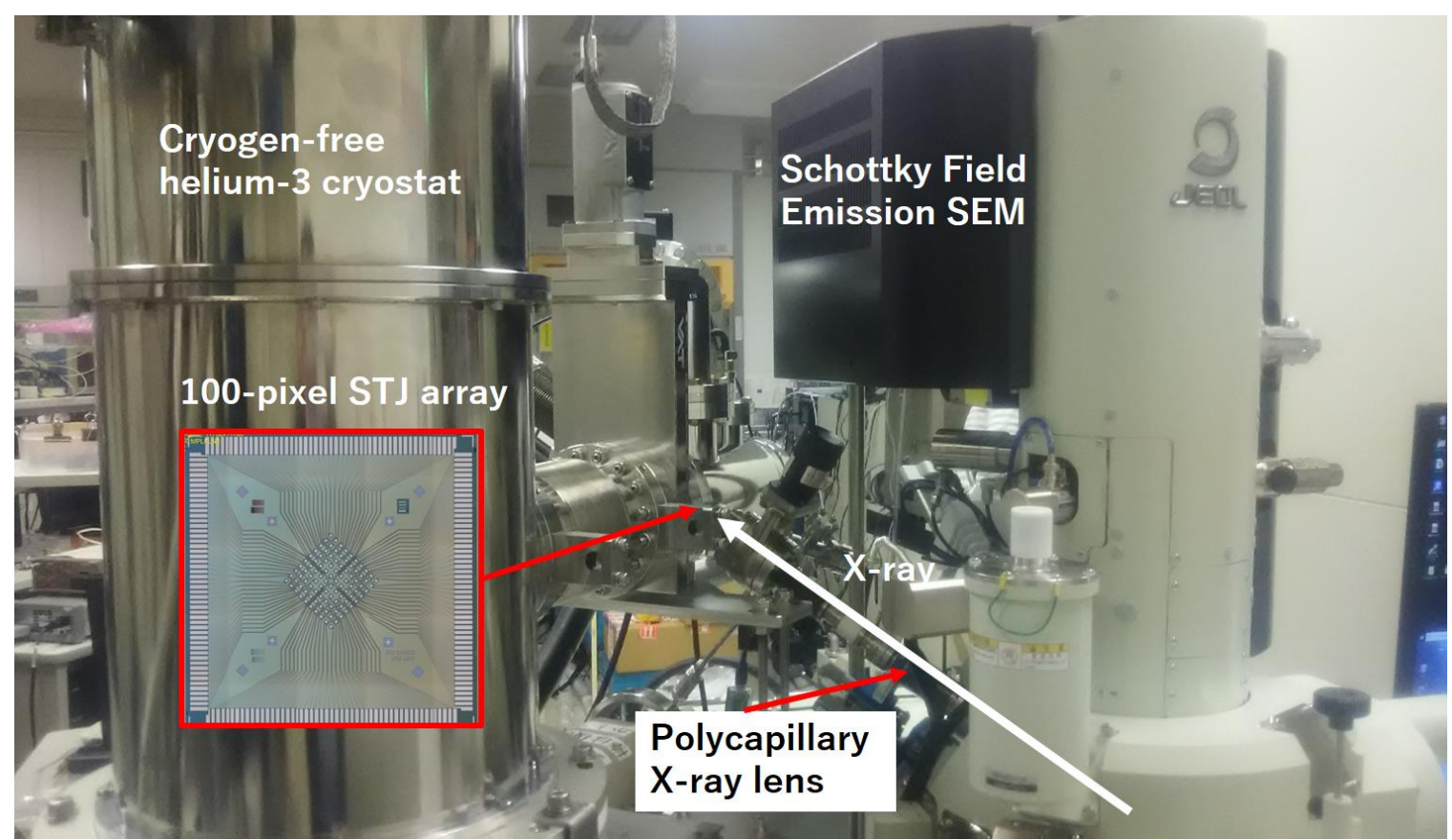

Figure 1. Picture of the SC-SEM 

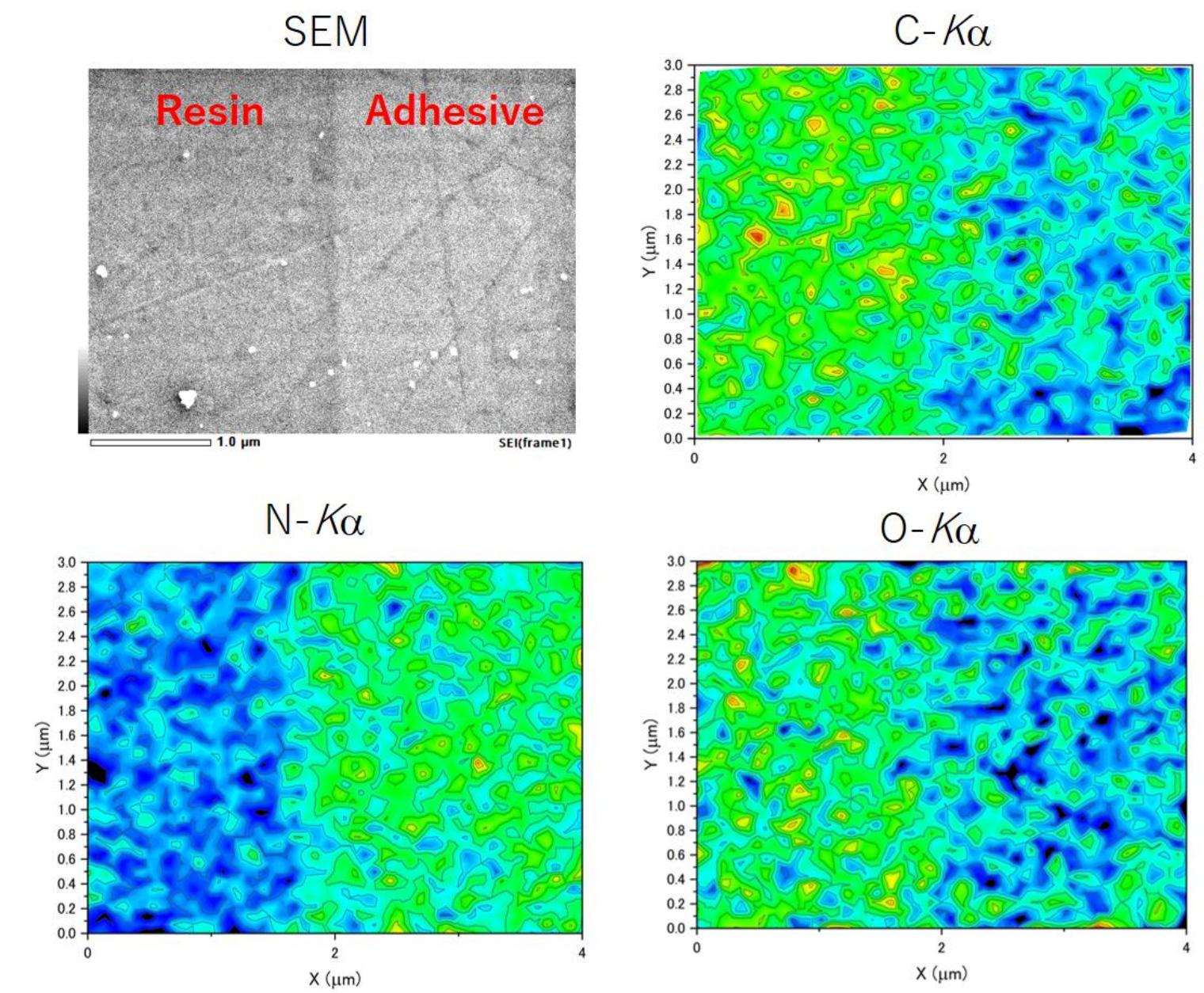

Figure 2. SEM and 2D elemental mapping images

\section{References}

[1] D.M. Schlosser, P. Lechner, G. Lutz, A. Niculae, H. Soltaua, L. Struder, R. Eckhardt, K. Hermenaua, G. Schaller, F. Schopper, O. Jaritschin, A. Liebel, A. Simsek, C. Fiorini, and A. Longoni, Nucl. Inst. Methods in Phys. 624 (2010) 270.

[2] H. Takahashi, T. Murano, M. Takakura, S. Asahina, M. Terauchi, M. Koike, T. Imazono. M. Koeda, and T. Nagano, IOP Conference Series: Materials Science and Engineering, 109 (2019) 012017.

[3] M. Terauchi and Y. Sato, Microscopy and Microanalysis 25 (2019) 1754.

[4] M. Ukibe, G. Fujii, S. Shiki, Y. Kitajima, and M. Ohkubo, J. Low. Temp. Phys. 184 (2016) 200.

[5] G. Fujii, M. Ukibe, S. Shiki, and M. Ohkubo, SIP-IMASM 2018 abstract booklet (2018) 23.

[6] G. Fujii, M. Ukibe, S. Shiki, and M. Ohkubo, X-ray spectrometry 46 (2017) 325.

[7] G. Fujii, M. Ukibe, S. Shiki, and M. Ohkubo, Microscopy and Microanalysis 25 (2019) 262. 